\title{
Classifier Combination for Face Localization in Color Images
}

\author{
Rachid Belaroussi, Lionel Prevost, and Maurice Milgram \\ LISIF Université Pierre et Marie Curie BC252, \\ 4 place Jussieu 75252 Paris cedex 05 France \\ rachid.belaroussi@gmail.com \\ \{lionel.prevost, maurice.milgram\}@lis.jussieu.fr
}

\begin{abstract}
We present a new method dedicated to the localization of faces in color images. It combines a connexionist model (auto-associative network), an ellipse model based on Generalized Hough Transform, a skin color model and an eyes detector that results in two features. A linear combination of the 3 first models is performed to eliminate most of non face regions. A connexionist combination of the four detectors response is performed on the remaining candidates. Given an input image, we compute a kind of probability map on it with a sliding window. The face position is then determined as the location of the absolute maximum over this map. Improvement of baseline detectors localization rates is clearly shown and results are very encouraging.
\end{abstract}

\section{Introduction}

Face detection in an image without any hypothesis is a tough task because of the high variability of the pattern to be detected [1]. As in many detection issues, it is almost impossible to define the opposite class, the non-face patterns, which drives researchers to choose the model-based approach. Solutions implemented in a large number of face detection applications (biometric, presence detection, visiophony, indexation, car driver detection, virtual reality, lips reading) start with simplifying the problem by making assumptions : fixed camera and known background, use of motion information [2], strong hypothesis on the face location, special background for an easy extraction of the silhouette or special lighting conditions (use of infra-red, for example). Face localization (the face is in the image and we want to know where) is not simpler without additional assumption.

We find here the two approach common in Pattern Recognition : structural and global. Structural approaches try to detect primitives of the face (eyes, mouth, nose, head edge) then combine the results using geometrical and radio metrical models, or constellations analysis [3]. Global approaches process a sub-image of the input image into a feature vector (momentum, projection, gray level, wavelet...). These approaches estimate the classifier parameters on a training set. In the global approach, parameters can be weights (neural networks) [4] or terms of a covariance matrix (statistical classifier). A choice is then to be made between the model approach and the discriminative one. A model does not require counter examples, which may seems an 
advantage but actually decreases classifier efficiency: generalization in a high dimension space ( 221 for $13 \times 17$ sub-images) is tough without knowing where are the vectors that might be confused. Another way is to design a combination of several detectors (classifiers). [5] did it to perform face detection and classifier combination has also been used in character [6], and face recognition [7].

In order to get the best of both worlds, our method makes co-operate holistic and structural approaches. Our face localization approach combines in a first stage (a prefilter resulting in regions of interest) an auto-associator network appearance based model, and an ellipse detector both based on the image gradient's direction, and a coarse skin color model in YCbCr color space. A second stage integrates these three detectors and two features related to the eyes as shown in fig. 1.

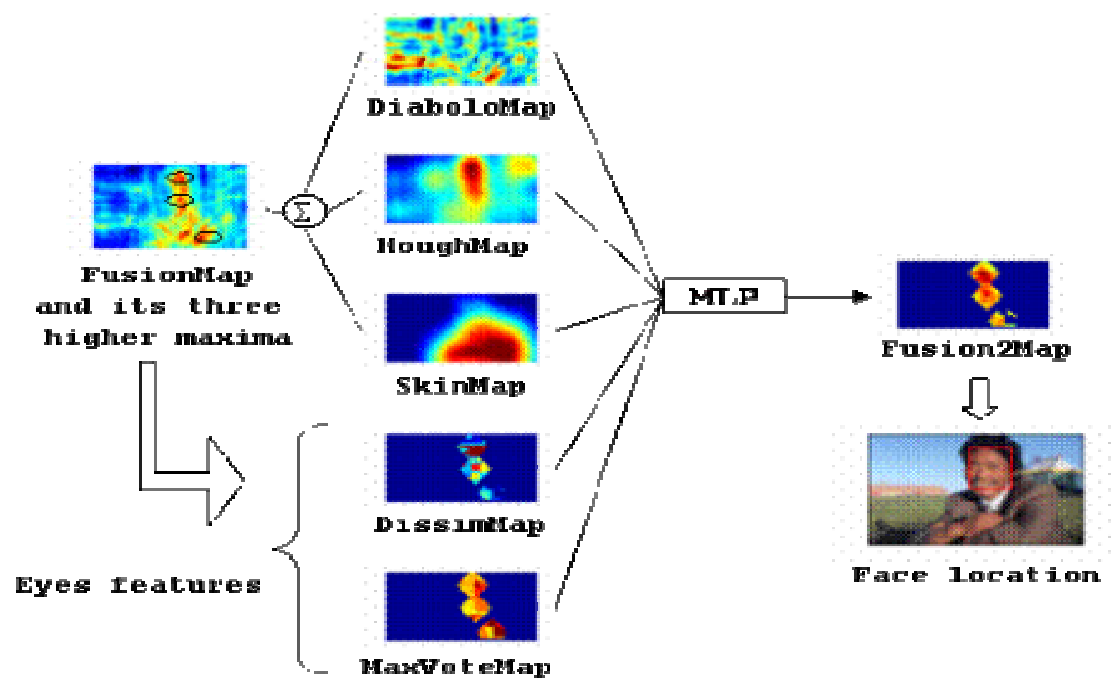

Fig. 1. Overview of the face localization system

Section 2 describes all these detectors, and their combination is detailed in Section 3. We present in Section 4 our experimental results and the benefits of the combination. The last section is devoted to conclusions and prospects.

\section{Basic Detectors}

\subsection{Edge Orientation Evaluation}

A part of the information of a face image lies in orientation of its edges. A huge advantage of the edge's orientation is its relative invariance to the skin tone. Three of the four detectors presented in the next sections use this information : the appearancebased model, the ellipse detector and the eyes detector. 
Evaluation of the gradient orientation on edge image requires a low-pass filtering of the image. Gradient field estimation uses Roberts masks, so that gradient magnitude on the $x$-axis and $y$-axis are computed as follow:

$$
I_{x}=I_{\text {filtered }} \otimes\left[\begin{array}{ll}
1 & -1
\end{array}\right] \text { and } I_{y}=I_{\text {filtered }} \otimes\left[\begin{array}{l}
1 \\
-1
\end{array}\right]
$$

Then, the gradient magnitude is thresholded. For the generalized Hough transform, a global threshold is applied over the whole input image. This threshold was optimized over 168 images and is equal to 12 . The thresholding for the neural network is defined over each $13 \times 17$ sub-windows of the input image, so that $20 \%$ of the pixels are then regarded as edges. Orientations of these edge pixels are then quantized on $\mathrm{N}=36$ values, except for the eyes detector which will be described in the last sub-section.

\subsection{Appearance-Based Model: The Auto-associative Multi-layer Perceptron (Diabolo)}

Pre-processing of training examples are described in [8]. Cosinus and sinus of the edge orientations inside an elliptical mask provide a 290 features vector for each face example. Processing of these examples is done with an auto-associator neural network, the so called "Diabolo" [9]. This network was successfully used for handwritten characters recognition [10], face detection [9] and compression [11]. It is trained to reconstruct an output identical to its input. It implements a specialized compression for its hidden layer has much less units than input or output does. So a non-face image should be badly compressed and the reconstruction error (square root of the mean square error between the input and the calculated output) would be higher than for a face image.

The neural network is trained using 1602 face images as a training set and training is stopped by cross-validation on 178 images. Weight and bias values are updated according to gradient descent with adaptive learning rate. After an exhaustive search we found that for 290 inputs (corresponding to a $17 \times 13$ retina) the optimal number of hidden cells is 18 . The gray level image is scanned at the resolution corresponding to the size of the face with a $13 \times 17$ sliding retina, and at each position of the image a reconstruction error is calculated. An array of reconstruction errors is calculated, we will referre to it as DiaboloMap.

\subsection{Ellipse Detector Based on Generalized Hough Transform}

Orientation of the gradient over the whole gray level image is then determined. Then, a Generalized Hough Transform (GHT) is performed on the resulting orientation map. Faces are modeled as vertical ellipses with a specific eccentricity so we can build up a lookup table to cast votes from each edge pixel, knowing its gradient orientation [8]. It provides a vote array which maximum correspond in the image to the position most likely to be the center of an ellipse with a horizontal minor axis $a=8$, and a vertical major axis $b=10$. The vote map is scanned using a $13 \times 17$ mexican hat which provides a new score map used to defined face location, we will referred to it as HoughMap (see fig. 2). 

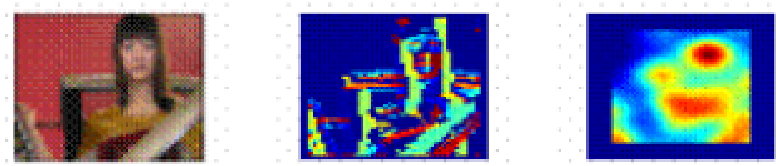

Fig. 2. Original image, gradient orientation of the edge and HoughMap

\subsection{Skin Color Detector}

Independently of gradient's orientation information, a coarse skin detection has been implemented in the YCbCr color space [12]. Our coarse skin color filter is defined by $\mathrm{Cb} \in\left[\begin{array}{ll}105 & 130\end{array}\right]$ and $\mathrm{Cr} \in\left[\begin{array}{ll}135 & 160\end{array}\right]$. These thresholds were experimentally tuned using images with people.

This skin detector is coarse [13] and in some case no skin at all is filtered but the combination of the detectors enables us to use a simple model. At each point of the image, we compute proportion of skin-color pixels inside the $13 \times 17$ neighbor. The result map is called SkinMap.

\subsection{Eyes Detector}

A global transformation, referred to as Chinese Transform (CT) [14], which input is the gradient orientations of the edge pixels of the image, provides as output a cumulative arrays (so called VOTE) that gives information on the centers of the eyes, and the size of a rectangular window around each eye. True eyes location correspond fairly well to the pixels with maximum number of votes. This maximum constitutes a first eyes feature, and its use is explained in the next section.

The latter technique provides a robust but not very precise localization of the eyes. A refinement tool is required to increase detection precision according to the vertical axis. The position of the eye center corresponds to some local minimum of the vertical profile. The profile is as usual the mean value of pixel values calculated for each line in the sub-window. Several local minimum are sometimes detected and are due to the eyebrows and/or the shade under the eye. As the disk formed by the pupil is darker than its environment (even for dark peoples) it corresponds to the profile global minimum.

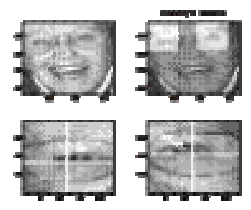

(a) $\mathrm{D}=0.019$
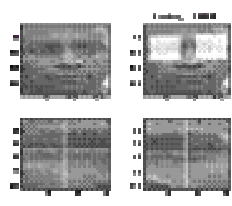

(b) $\mathrm{D}=0.017$

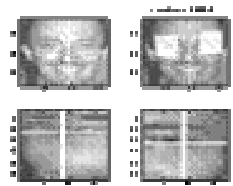

(c) $\mathrm{D}=0.025$

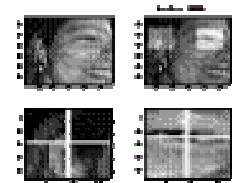

(d) $\mathrm{D}=0.22$

Fig. 3. Dissimilarity values for good (a,b,c) and false (d) detections 
A validation step simply compares images of both eyes. The dissimilarity (after vertical symmetry) is calculated by normalizing grayscale and size of the two subwindows to be compared. Then we apply a vertical symmetry (left/right flip) to the second image. The dissimilarity is the Euclidean distance between these 2 normalized and flipped windows (see fig. 3). This dissimilarity constitute the last of five face features, yielding the DissimilMap as we will see in section 3.

\section{Neural Combination}

These five sources of information are combined for face localization purpose. In a first stage the first three (appearance-based model, ellipse detector and skin color detector) are linearly combined to eliminate most of non-face windows. A second stage fusions the five detectors using a neural network in order to localize the face.

We have implemented three detectors for a color image, which result in three maps : DiaboloMap, HoughMap, and SkinMap. Each detector map is linearly adjusted onto $\left[\begin{array}{ll}-1 & 1\end{array}\right]$. We will denote these normalized maps as D, H, and S. Using the three detectors, a pixel $(\mathrm{i}, \mathrm{j})$ in the original image is then featured by $I_{i, j}=\left[\begin{array}{lll}H_{i, j} & D_{i, j} & S_{i, j}\end{array}\right]$. Examples extracted from 100 images of the diabolo's cross-validation set are used to learn linear combination parameters using a gradient descent stopped by cross validation :

$$
\text { FusionMap }_{i, j}=a \cdot H_{i, j}-b \cdot D_{i, j}+c \cdot S_{i, j}
$$

with $a=0.2280, b=0.2620, c=0.1229$.

We can notice that the weight of the skin detector is smaller than Hough and diabolo detectors ones. This linear combination is used as a pre-filter for the eye features calculation. Each maxima of the FusionMap correspond to a sub-image in the original image. Face candidates are the windows close enough to three higher maxima (an example is given in fig. 4). We call them "face candidate windows". A window is "close enough" to a maxima when it overlap the corresponding sub-image by more than $60 \%$.

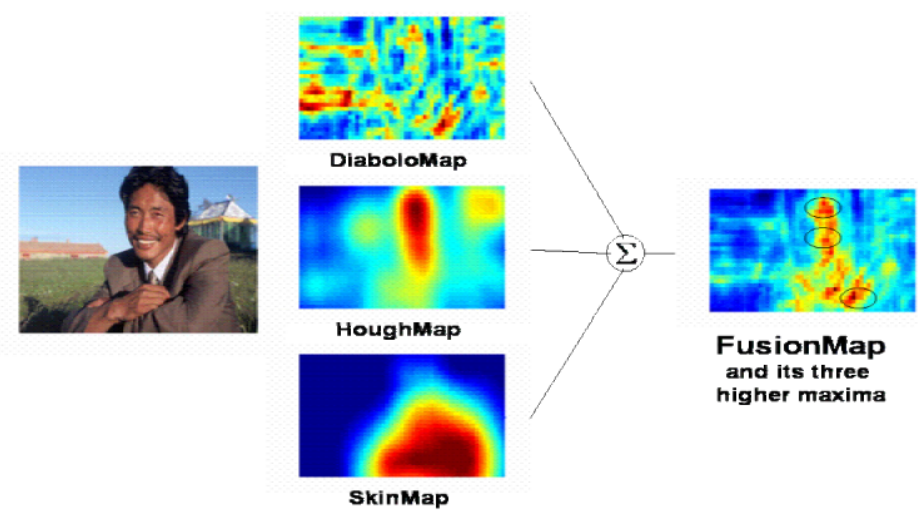

Fig. 4. Overview of the pre-filtering stage 
The two eye features (dissimilarity and maximum number of votes in the eye VOTE array) are calculated over these «face candidate windows » resulting in two arrays, DissimMap and MaxVoteMap, linearly adjusted onto $\left[\begin{array}{ll}-1 & 1\end{array}\right]$ (see fig. 5).

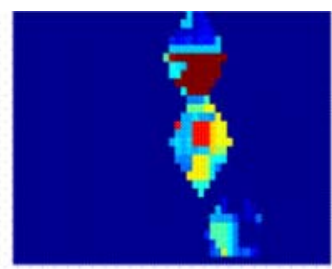

DissimMap

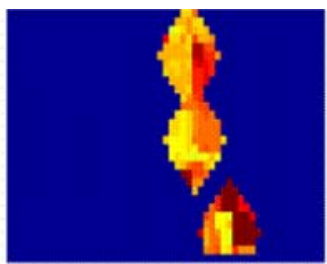

MaxVoteMap

Fig. 5. Eye features calculated on face candidates resulting from the pre-filter

On our test database of 1352 images, the linear combination is calculated over 3001558 sub-windows, and only 271896 sub-images are submitted to the Chinese Transform. So, the first stage eliminate $\mathbf{9 1 \%}$ of all possible windows.

This process is implemented over 155 images of the training database described earlier providing five maps used to train, with a gradient descent stopped by cross validation, a multilayer perceptron (MLP). Its architecture consists of 5 inputs, 4 hidden neurons (with a sigmoid activation function) and one output neuron (with a linear activation function).

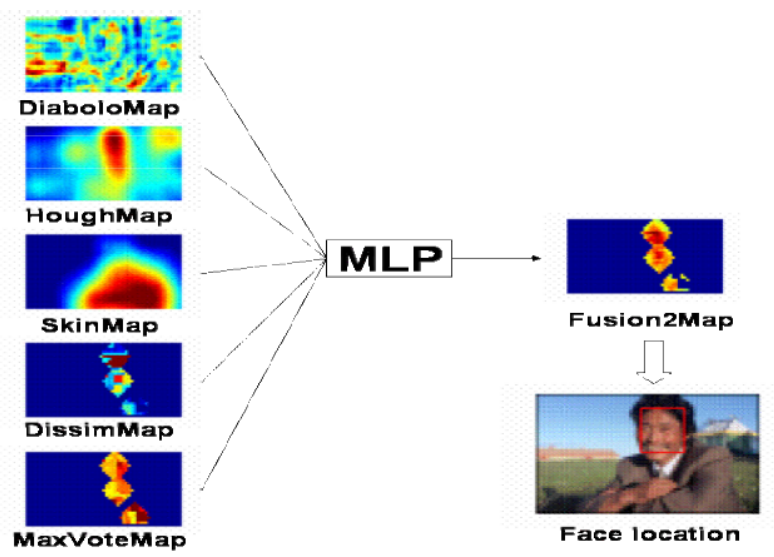

Fig. 6. Face location correspond to Fusion2Map maximum

The MLP is trained to output +1 if the input image is a face, and -1 if it's a non face.

MLP response over the complete image is called Fusion2Map, and the maximum of this map defines face location in the original image as shown in fig. 6 . 


\section{Face Localization Performances Evaluation}

Combining multiple sources greatly improves localization performances [15]. To determine face location, the input image is scanned (first by the pre-filter then by the MLP) and the location of the face is defined as the maximum of the resulting map Fusion2Map. We use the ECU face database [16] which is composed of color images (set 1), and two corresponding ground truth : one for the faces (set 2) and one for the skin (set 3). This database includes faces with various poses and skin tones.

Our test set is made of 1352 images (non overlapping with the training and crossvalidation corpus) containing only one face, to evaluate localization rate. For each image, face's size is supposed to be known, which enables us to apply a $13 \times 17$ window sliding strategy, this knowledge is equivalent to knowing the distance between the camera and the person to be localized. Each image of the test set is first resized so that the face (defined by the ground truth) reaches the size $13 \times 17$. This size was chosen to respects faces aspect ratio, and is a good compromise between face's feature visibility (by human vision) and computational efforts.

A face is considered as correctly localized if the detection bounding box covers at least $60 \%$ of the area of the bounding box defined by the ground truth. To evaluate improvement brought by the combination of the 5 detectors, the proportion of pixels of the ground truth inside the detection (which is the location of the maximum for HoughMap and of the minimum for DiaboloMap) is calculated for each test image. Using the DiaboloMap minimum position alone, 656 faces are correctly located (proportion of good pixels greater than 0.6) in our test set (48.5\%). Using the maximum of HoughMap alone to define the location of the face, 903 faces are correctly detected $(67 \%)$.

After combination, 1197 faces (over the 1352 test images) are correctly detected which increases the detection rate to $\mathbf{8 8 . 5 \%}$. The combination of the five detectors decreases the error rate of more than $50 \%$.

\section{Conclusion and Prospects}

This communication aimed to present a significant contribution to the face localization task. We have presented five different detectors: skin color, autoassociative multi-layer perceptron, ellipse Hough Transform, and two eye features. A linear combination of the first three feature eliminates most of face candidate, and activates the eye detector. The five detectors are combined using a MLP and an awesome improvement of localization rate.

Several improvements are in progress: more sophisticated skin color models like ellipsoidal thresholding, Gaussian density functions [17] or mixture of Gaussians [18]. Note that the Chinese Transform is also useful in other contexts like detection of axial symmetries or the localization of patterns [14]. Our experimental results show that to solve the problem arising from tilted or slanted heads, a 3D pose detection of the head is necessary; this detection should be done without the localization of the eyes, with an holistic algorithm. Our team already test such an approach for face pose estimation which gives promising results. 


\section{References}

1. Yang, M.-H, Kriegman, D., Ahuja, N.: Detecting Faces in Images: A Survey, IEEE Trans. Pattern Analysis and Machine Intelligence, vol. 24, no. 1, (2002), 34-58

2. Verma, R.C., Schmid, C., Mikolajczyk, K.:Face Detection and Tracking in a Video by Propagating Detection Probabilities, IEEE Trans. PAMI, vol.25, no 10, Oct. 2003

3. Bileschi, S.M., Heisele, B.: Advances in Component Based Face Detection, IEEE International Workshop on Analysis and Modeling of Face and Gestures (2003)

4. Garcia, C., Delakis, M.: Convolutional Face Finder: A Neural Architecture for Fast and Robust Face Detection IEEE Trans. PAMI, vol. 26, no. 11, Nov. 2004

5. Froba, B., Zink, W.: On the combination of different template strategies for fast face detection, Multiple Classifier Systems, (2001) 418-428

6. Rahman, A.F., Fairhust, M.C.: Multiple classifier decision combination strategies for character recognition: a review, International Journal on Document Analysis and Recognition, (5) (2003) 166-194

7. Czyz, J., Kittler, J., Vandendorpe, L.: Combining face verification experts, International Conference on Pattern Recognition, vol 2, (2002) 28-31

8. Belaroussi, R., Prevost, L., Milgram, M.: Combining model-based classifiers for face localization, to appear in Proc. of IAPR Conf. on Machine Vision \& Application (2005)

9. Féraud, R., Bernier, O., Viallet, J., Collobert, M.: A Fast and Accurate Face Detector Based on Neural Networks, IEEE Trans.PAMI, vol. 23, no. 1, (2002) 42-53

10. Schwenk, H., Milgram, M.: Transformation invariant auto-association with application to handwritten character recognition, Neural Information Processing Systems 7, (1995) 991-998

11. DeMers, D. and Cottrell, G.: Non-linear dimensionality reduction. Neural Information Processing Systems 5, (1993) 580-587

12. Chai, D., Nang, K.N.: Locating facial region of a head-and-shoulders color image. International conference on Automatic Face and Gesture Recognition, (1998) 124-129

13. $\mathrm{Hu}$, M., Worrall, S., Sadka, A.H., Kondoz, A.M.: Automatic scalable face model design for 2D model-based video coding, Signal Processing: Image Communication, vol. 19, (2004) 421-436

14. Milgram, M., Belaroussi, R., Prevost., L.: Multi-stage combination of geometric and colorimetric detectors for eyes localization. to appear in Proc. of ICIAP 2005

15. Prevost, L., Milgram, M.: Automatic Allograph Selection and Multiple Expert Classification for Totally Unconstrained Handwritten Character Recognition, International Conference on Pattern Recognition, vol 1, (1998) 381-383

16. Phung, S. L., Bouzerdoum, A., Chai, D.: Skin segmentation using color pixel classification: Analysis and comparison, IEEE Trans. PAMI, vol. 27, no. 1, (2005) 148-154

17. Yang, M.-H, Ahuja, N.: Detecting human faces in color images, Proceedings Of IEEE International Conference on Image Processing, vol. 1, (1998) 127-130

18. McKenna, S.J., Gong, S., Raja, Y.: Modeling facial color and identity with gaussian mixtures, Pattern Recognition, vol 31, no.12, (1998) 1883-1892 